\title{
RATIONAL TILINGS BY $n$-DIMENSIONAL CROSSES. II
}

\author{
S. SZABÓ
}

\begin{abstract}
The union of translates of a closed unit $n$-dimensional cube whose edges are parallel to the coordinate unit vectors $\mathbf{e}_{1}, \ldots, \mathbf{e}_{n}$ and whose centers are $i \mathbf{e}_{j},|i| \leq k, 1 \leq j \leq n$, is called a $(k, n)$-cross. A system of translates of a $(k, n)$-cross is called an integer (a rational) lattice tiling if its union is $n$-space and the interiors of its elements are disjoint, the translates form a lattice and each translation vector of the lattice has integer (rational) coordinates. In this paper we shall continue the examination of rational cross tilings begun in [2], constructing rational lattice tilings by crosses that have noninteger coordinates on several axes.
\end{abstract}

1. Introduction. Denote by $\mathbf{e}_{1}, \ldots, \mathbf{e}_{n}$ the coordinate unit vectors and denote by $C_{P}$ the $n$-dimensional closed unit cube whose edges are parallel to $\mathbf{e}_{1}, \ldots, \mathbf{e}_{n}$ and whose center is $P$. Let $Z$ be the set of integers and let $T$ be a finite subset of $Z^{n}$. Let $\tau_{O}$ be the union of the $C_{P}$ 's, where $\overrightarrow{O P} \in\left\{t_{1} \mathbf{e}_{1}+\cdots+t_{n} \mathbf{e}_{n}:\left(t_{1}, \ldots, t_{n}\right) \in T\right\}$. We use the notation $\left(\tau_{O}, \mathbf{L}\right):=\left\{\tau_{P}: \overrightarrow{O P} \in \mathbf{L}\right\}$ for an arbitrary vector set $\mathbf{L}$. If the system $\left(\tau_{O}, \mathbf{L}\right)$ covers $n$-space and if the interiors of its elements are disjoint, then we say that $\left(\tau_{O}, \mathbf{L}\right)$ is a tiling. If every coordinate of each vector of $\mathbf{L}$ is an integer (is rational), then the tiling is called an integer (a rational) tiling.

Let $q_{1}, \ldots, q_{n}$ be fixed positive integers and let $\mathbf{X}$ be the lattice spanned by the vectors $\left(1 / q_{1}\right) \mathbf{e}_{1}, \ldots,\left(1 / q_{n}\right) \mathbf{e}_{n}$.

If we select $T \subseteq Z^{n}$ such that

$$
T=\{(0, \ldots, 0),(i, 0, \ldots, 0), \ldots,(0, \ldots, 0, i): 1 \leq|i| \leq k\},
$$

then the $\tau_{O}$ that belongs to $T$ is a $(k, n)$-cross. It consists of $2 k n+1$ cubes; a central cube together with $2 n$ arms of length $k$. The set $\tau_{O}$ belonging to

$$
T=\{(0, \ldots, 0),(i, 0, \ldots, 0), \ldots,(0, \ldots, 0, i): 1 \leq i \leq k\}
$$

is called a $(k, n)$-semicross. It consists of $k n+1$ cubes; a corner cube together with $n$ arms of length $k$.

In [2] we constructed noninteger lattice tilings by $(k, n)$-crosses and semicrosses for certain $k$ and $n$. In these tilings only one fixed coordinate of the translation vectors was a noninteger. The purpose of this paper is to construct more general types of noninteger lattice tilings by $(k, n)$-crosses and semicrosses, namely rational lattice tilings in which some lattice vectors have several noninteger coordinates. The number of noninteger coordinates will depend on the prime factorization of the numbers $2 k n+1$ and $k n+1$ respectively.

Received by the editors April 27, 1983.

1980 Mathematics Subject Classification. Primary 20K01; Secondary 10 E30.

Key words and phrases. Factorization splitting and partition of abelian groups, crosses, semicrosses, lattice tiling. 
We shall use algebraic techniques. Let $G$ be an abelian group which is written additively and let $H, A_{1}, \ldots, A_{s}$ be subsets of $G$ and assume that each of them contains the zero element. If each element $h \in H$ is uniquely expressible in the form $h=a_{1}+\cdots+a_{s}, a_{1} \in A_{1}, \ldots, a_{s} \in A_{s}$, and each sum $a_{1}+\cdots+a_{s}$ is in $H$, then we write $H=A_{1}+\cdots+A_{s}$ and speak of a factorization of $H$. If each element $h \in H$ is uniquely expressible in the form $h=t_{1} g_{1}+\cdots+t_{n} g_{n},\left(t_{1}, \ldots, t_{n}\right) \in T$, where $g_{1}, \ldots, g_{n}$ are fixed elements of $G$, then we shall write $H=T\left(g_{1}, \ldots, g_{n}\right)^{*}$. (We may think of the multiplication of the "matrix" $T$ by the "vector" $\left(g_{1}, \ldots, g_{n}\right)^{*}$.) If $g$ is a nonzero element of $G$ and has order at least $q$, then we shall denote the set $\{0, g, 2 g, \ldots,(q-1) g\}$ by $[g]_{q}$. The subscript $q$ denotes the cardinality of the set.

2. General results. We wish to use the following results from [2].

Theorem $2.1\left[\mathbf{2}\right.$, TheOREM 2.1]. (1) There exists a tiling $\left(\tau_{O}, \mathbf{L}\right), \mathbf{L} \subseteq \mathbf{X}$ if and only if there exists an abelian group $G$ which is factorizable in the form

$$
G=T\left(q_{1} g_{1}, \ldots, q_{n} g_{n}\right)^{*}+\left[g_{1}\right]_{q_{1}}+\cdots+\left[g_{n}\right]_{q_{n}}+H,
$$

where $g_{1}, \ldots, g_{n}$ generate $G$ and $H$ is a subset of $G$.

(2) $\mathbf{L}=H \varphi^{-1}$, where $\varphi: \mathbf{X} \rightarrow G$ is given by

$$
\left(\left(z_{1} / q_{1}\right) \mathbf{e}_{1}+\cdots+\left(z_{n} / q_{n}\right) \mathbf{e}_{n}\right) \varphi=z_{1} g_{1}+\cdots+z_{n} g_{n} ; \quad z_{1}, \ldots, z_{n} \in Z .
$$

(3) The set $\mathbf{L}$ is a lattice if and only if $H$ is a subgroup of $G$, denoted $H \triangleleft G$.

REMARK 2.1. If $\mathbf{L}$ is a rational lattice, then there are positive integers $q_{1}, \ldots, q_{n}$ $\in Z$ such that $\mathbf{L} \subseteq \mathbf{X}$, where $\mathbf{X}$ is spanned by $\mathbf{e}_{1} / q_{1}, \ldots, \mathbf{e}_{n} / q_{n}$. Indeed, if $\mathbf{L}$ is spanned by $\mathbf{l}_{1}, \ldots, \mathbf{l}_{n}$ and if $\mathbf{l}_{i}=\sum\left(p_{i j} / q_{i j}\right) \mathbf{e}_{j}, p_{i j}, q_{i j} \in Z, q_{i j}>0$, then $q_{j}$ can be the least common multiple of $q_{1 j}, \ldots, q_{n j}$.

REMARK 2.2. When $\left(\tau_{O}, \mathbf{L}\right)$ is a lattice tiling, we may assume that $H=\{0\}$ in (2.1) since if $H \triangleleft G$ we can replace $G$ by the factor group $G / H$.

The next theorem generalizes the third statement in Theorem 2.1 in [2]. The symbol $\sum_{j \neq i}$ is short for $\sum_{j=i, j \neq i}^{n}$.

THEOREM 2.2. Let $\left(\tau_{O}, \mathbf{L}\right), \mathbf{L} \subseteq \mathbf{X}$ be a tiling and assume that $\mathbf{e}_{1}, \ldots, \mathbf{e}_{n} \in$ $T\left(\mathbf{e}_{1}, \ldots, \mathbf{e}_{n}\right)^{*}$. The ith coordinate of each vector of $\mathbf{L}$ is an integer if and only if the set

$$
S_{i}=T\left(q_{1} g_{1}, \ldots, q_{n} g_{n}\right)^{*}+H+\sum_{j \neq i}\left[g_{j}\right]_{q_{j}}
$$

in the factorization (2.1) is a subgroup of $G$.

PROOF. For simplicity we shall prove only the case $i=1$. Assume that the set $S_{1}$ is a subgroup of $G$. We shall prove that if $\mathbf{l} \in \mathbf{L}$, then the first coordinate of $\mathbf{l}$ is an integer.

Since $\left(\tau_{O}, \mathbf{L}\right)$ is a tiling and $\mathbf{L} \subseteq \mathbf{X}$, we see that $-\mathbf{l}$ is uniquely expressible in the form $-\mathbf{l}=\left(z_{1} / q_{1}\right) \mathbf{e}_{1}+\cdots+\left(z_{n} / q_{n}\right) \mathbf{e}_{n}, z_{1}, \ldots, z_{n} \in Z$. Let $u_{i}, v_{i}$ be integers such that $z_{i}=u_{i} q_{i}+v_{i}, 0 \leq v_{i}<q_{i}$. Thus we have

$$
-\mathbf{l}=\sum_{j=1}^{n} u_{j} \mathbf{e}_{j}+\sum_{j=1}^{n}\left(v_{j} / q_{j}\right) \mathbf{e}_{j} .
$$


Applying the homomorphism $\varphi$ defined by (2.2), we obtain

$$
(-\mathbf{l}) \varphi=\sum_{j=1}^{n} u_{j} q_{j} g_{j}+\sum_{j=1}^{n} v_{j} g_{j}
$$

We know that $\mathbf{e}_{1}, \ldots, \mathbf{e}_{n} \in T\left(\mathbf{e}_{1}, \ldots, \mathbf{e}_{n}\right)^{*}$, hence $q_{1} g_{1}, \ldots, q_{n} g_{n} \in T\left(q_{1} g_{q}, \ldots, q_{n} g_{n}\right)^{*}$, and also that $(\mathbf{l}) \varphi \in H$. Denote $(\mathbf{l} \varphi)$ by $h$. Then

$$
0=\left(h+\sum_{j=1}^{n} u_{j} q_{j} g_{j}+\sum_{j=1}^{n} v_{j} g_{j}\right) \in S_{1} .
$$

Since $h+\sum_{j=1}^{n} u_{j} q_{j} g_{j}+\sum_{j=2}^{n} v_{j} g_{j}$ is also in $S_{1}$, the difference between these two sums, namely $v_{1} g_{1}$, is also in $S_{1}$. Since the sum (2.1) is direct, $v_{1}=0$, and it follows from (2.4) that the first coordinate of $l$ is an integer.

Assume that the first coordinate of each vector of $\mathbf{L}$ is an integer: We shall show that $S_{1} \triangleleft G$. Let $\mathbf{t}, \mathbf{t}^{\prime} \in T\left(\mathbf{e}_{1}, \ldots, \mathbf{e}_{n}\right)^{*}, \mathbf{l}, \mathbf{l}^{\prime} \in \mathbf{L}$, and $0 \leq v_{j}, v_{j}^{\prime}<q_{j}, 2 \leq j \leq n$. The first coordinate of the vector

$$
\left(\mathbf{t}+\mathbf{l}+\sum_{j=2}^{n}\left(v_{j} / q_{j}\right) \mathbf{e}_{j}\right)-\left(\mathbf{t}^{\prime}+\mathbf{l}^{\prime}+\sum_{j=2}^{n}\left(v_{j}^{\prime} / q_{j}\right) \mathbf{e}_{j}\right)
$$

is an integer. Since $\left(\tau_{O}, \mathbf{L}\right)$ is a tiling and $\mathbf{L} \subseteq \mathbf{X}$, there exist $\mathbf{t}^{\prime \prime} \in T\left(\mathbf{e}_{1}, \ldots, \mathbf{e}_{n}\right)^{*}$, $\mathbf{l}^{\prime \prime} \in \mathbf{L}$ and $0 \leq v_{j}^{\prime \prime}<q_{j}, 1 \leq j \leq n$, such that the vector $(2.5)$ equals $\mathbf{t}^{\prime \prime}+\mathbf{l}^{\prime \prime}+$ $\sum_{j=1}^{n}\left(v_{j}^{\prime \prime} / q_{j}\right) \mathbf{e}_{j}$. thus $v_{1}^{\prime \prime}=0$. Application of the homomorphism $\varphi$ shows that $S_{1} \triangleleft G$.

REMARK 2.3. The assumption $\mathbf{e}_{1}, \ldots, \mathbf{e}_{n} \in T\left(\mathbf{e}_{1}, \ldots, \mathbf{e}_{n}\right)^{*}$ cannot be removed, as the following 2 -dimensional tiling illustrates. Let $\mathbf{X}$ and $\mathbf{L}$ be the lattice spanned by $(1 / 2) \mathbf{e}_{1}, \mathbf{e}_{2}$ and $2 \mathbf{e}_{1},(-1 / 2) \mathbf{e}_{1}+\mathbf{e}_{2}$ respectively, and let $T=\{(0,0),(1,0)\}$. Obviously, $\left(\tau_{O}, \mathbf{L}\right)$ is a lattice tiling, that is, $\mathbf{X}=\mathbf{T}\left(\mathbf{e}_{1}, \mathbf{e}_{2}\right)^{*}+\left[(1 / 2) \mathbf{e}_{1}\right]_{2}+\mathbf{L}$ is a factorization of $\mathbf{X}$. Let $G$ be a cyclic group of order 4 and let $g$ be one of its generators. If $g_{1}=g_{2}=g$ and $\varphi: \mathbf{X} \rightarrow G$ is defined by $\left(\left(z_{1} / 2\right) \mathbf{e}_{1}+z_{2} \mathbf{e}_{2}\right) \varphi=$ $z_{1} g_{1}+z_{2} g_{2}, z_{1}, z_{2} \in Z$, then we have the factorization $G=T\left(2 g_{1}, g_{2}\right)^{*}+\left[g_{1}\right]_{2}$. Now the lattice $\mathbf{L}$ is not an integer lattice but

$$
T\left(2 g_{1}, g_{2}\right)^{*}=\left\{0 \cdot 2 g_{1}+0 \cdot g_{2}, 1 \cdot 2 g_{1}+0 \cdot g_{2}\right\}=\{0,2 g\} \triangleleft G .
$$

THEOREM 2.3. If there is an abelian group $G$ which is factorizable in the form (2.1) and $q_{1}>1, \ldots, q_{s}>1, s<n$, and $g_{n}$ generates $G$, then there is a lattice tiling $\left(\tau_{O}, \mathbf{L}\right), \mathbf{L} \subseteq \mathbf{X}$ such that $\mathbf{L}$ has a vector whose first $s$ coordinates are not integers.

Proof. According to Theorem 2.1 the system $\left(\tau_{O}, H \varphi^{-1}\right)$ is a tiling, where $\varphi: \mathbf{X} \rightarrow G$ is given by (2.2). There exists $a_{i} \in Z$ such that $g_{i}=a_{i} g_{n}, 1 \leq i \leq s$, since $g_{n}$ generates $G$. The vectors $\left(-1 / q_{i}\right) \mathbf{e}_{i}+\left(a_{i} / q_{n}\right) \mathbf{e}_{n}$ are elements of $H \varphi^{-1}$, because

$$
\left(\left(-1 / q_{i}\right) \mathbf{e}_{i}+\left(a_{i} / q_{n}\right) \mathbf{e}_{n}\right) \varphi=\left(-1 / q_{i}\right) \mathbf{e}_{i} \varphi+\left(a_{i} / q_{n}\right) \mathbf{e}_{n} \varphi=-g_{i}+a_{i} g_{n}=0 .
$$

Hence the sum of these vectors is an element of $H \varphi^{-1}$ as well and its first $s$ coordinates are not integers. 
REMARK 2.4. The referee has pointed out that if in a lattice there exists for each $i, 1 \leq i \leq n$, a lattice vector whose $i$ th coordinate is not an integer, then it does not follow that there exists a lattice vector all of whose coordinates are not integers. Indeed, let $n$ be an odd number and consider the lattice spanned by the vectors $(1 / 2)\left(\mathbf{e}_{1}+\cdots+\mathbf{e}_{n}\right)+(1 / 2) \mathbf{e}_{i}, 1 \leq i \leq n$.

3. Tilings by crosses and semicrosses. In this section we apply the preceding theory to two special sets $T$, the $(k, n)$-cross and $(k, n)$-semicross.

The next lemma plays an important part in the proof of the following theorem.

LEMmA 3.1. If $H, A, B$ are subsets of an abelian group, $H=A+B$ is a factorization and $A=\{0\} \cup A^{\prime} \cup\left(-A^{\prime}\right)$ and $B=\{0\} \cup B^{\prime} \cup\left(-B^{\prime}\right)$ are partitions, then there exists a partition $H=\{0\} \cup H^{\prime} \cup\left(-H^{\prime}\right)$.

Proof. Let $H^{\prime}=A^{\prime} \cup B^{\prime} \cup\left(A^{\prime}+B^{\prime}\right) \cup\left(A^{\prime}-B^{\prime}\right)$.

THEOREM 3.1. If $2 n+1$ is the product of $s$ primes, then there exists a rational lattice tiling by $(1, n)$-crosses in which one of the lattice vectors has $s-1$ noninteger coordinates.

PROOF. According to Theorem 2.3, it is sufficient to give integers $q_{1}, \ldots, q_{n}$, an abelian group $G$, and its factorization in the form

$$
G=\left\{0, q_{1} g_{1}, \ldots, q_{n} g_{n},-q_{1} g_{1}, \ldots,-q_{n} g_{n}\right\}+\left[g_{1}\right]_{q_{1}}+\cdots+\left[g_{n}\right]_{q_{n}},
$$

where $q_{1}>1, \ldots, q_{s-1}>1$, and $g_{n}$ generates $G$.

Let $q_{1}, \ldots, q_{n}, r_{1}, \ldots, r_{s}$ be integers such that $q_{s}=\cdots=q_{n}=1$, and the others are greater than one, and $r_{1} \cdots r_{s}=2 n+1$. Let $G$ be the cyclic group of order $(2 n+1) q_{1} \cdots q_{s-1}$ and let $g$ be one of its generators. Obviously, $r_{1}, \ldots, r_{s}$ are odd numbers. The factorizations

$$
\begin{gathered}
G=\left\{\left(-\left(r_{1}-1\right) / 2\right) g, \ldots,-g, 0, g, \ldots,\left(\left(r_{1}-1\right) / 2\right) g\right\}+\left[r_{1} g\right]_{q_{1}}+r_{1} q_{1} G, \\
r_{1} q_{1} G=r_{1} q_{1}\left(\left\{\left(-\left(r_{2}-1\right) / 2\right) g, \ldots,-g, 0, g, \ldots,\left(\left(r_{2}-1\right) / 2\right) g\right\}+\left[r_{2} g\right]_{q_{2}}+r_{2} q_{2} G\right),
\end{gathered}
$$

etc. show that there exists a factorization

$$
G=V_{1}+\cdots+V_{s}+\left[g_{1}\right]_{q_{1}}+\cdots+\left[g_{s-1}\right]_{q_{s-1}},
$$

where $g_{1}=r_{1} g, g_{i}=r_{1} \cdots r_{i} q_{1} \cdots q_{i-1} g, 2 \leq i \leq s-1$, and

$$
\begin{gathered}
V_{1}=\left\{\left(-r\left(r_{1}-1\right) / 2\right) g, \ldots,-g, 0, g, \ldots,\left(\left(r_{1}-1\right) / 2\right) g\right\} \\
V_{i+1}=q_{i}\left\{\left(-\left(r_{i+1}-1\right) / 2\right) g_{i}, \ldots,-g_{i}, 0, g_{i}, \ldots,\left(\left(r_{i+1}-1\right) / 2\right) g_{i}\right\}, \quad 1 \leq i \leq s-1 .
\end{gathered}
$$

Obviously, $q_{i} g_{i} \in V_{i+1}, 1 \leq i \leq s-1$. By Lemma 3.1 we conclude that there exists a partition $V_{1}+\cdots+V_{s}=\{0\} \cup V \cup(-V)$. Since $\left|V_{1}+\cdots+V_{s}\right|=r_{1} \cdots r_{s}=2 n+1=$ $2|V|+1$, we can denote the elements of $V \backslash\left\{q_{1} g_{1}, \ldots, q_{s-1} g_{s-1}\right\}$ by $g_{s}, \ldots, g_{n}$. So

$$
V_{1}+\cdots+V_{s}=\left\{0, q_{1} g_{1}, \ldots, q_{n} g_{n},-q_{1} g_{1}, \ldots,-q_{n} g_{n}\right\},
$$

that is, $G$ is factorizable in the form (3.1). Since $g \in V_{1}$, we may assume that $g_{n}=g$. Thus $T\left(q_{1} g_{1}, \ldots, q_{n} g_{n}\right)^{*}$ generates $G$. It is then a simple matter to apply Theorem 2.2.

A similar method can be used to prove the following theorem. 
THEOREM 3.2. If $n+1$ is the product of $s$ primes, then there exists a rational lattice tiling by $(1, n)$-semicrosses in which one of the lattice vectors has $s-1$ noninteger coordinates.

We shall illustrate our method in the case of the $(1,7)$-semicrosses. We have $r_{1}=r_{2}=r_{3}=2$, and choose $q_{1}=q_{2}=3, q_{3}=\cdots=q_{7}=1$, and so let $G$ be the cyclic group of order $r_{1} r_{2} r_{3} q_{1} q_{2}=72$ with $72 g=0$ as its defining relation. Consider the factorization

$$
\begin{aligned}
G & =\{0, g\}+\{0,2 g, 4 g\}+\{0,6 g\}+\{0,12 g, 24 g\}+\{0,36 g\} \\
& =\{0,3 \cdot 2 g, 3 \cdot 12 g, 7 g, 37 g, 42 g, 43 g, g\}+[2 g]_{3}+[12 g]_{3} .
\end{aligned}
$$

According to Theorem 2.1, there exists a lattice tiling by $(1,7)$-semicrosses. The vectors $-(1 / 3) \mathbf{e}_{1}+2 \mathbf{e}_{7},-(1 / 3) \mathbf{e}_{2}+12 \mathbf{e}_{7},-\mathbf{e}_{3}+7 \mathbf{e}_{7},-\mathbf{e}_{4}+37 \mathbf{e}_{7},-\mathbf{e}_{5}+42 \mathbf{e}_{7}$, $-\mathbf{e}_{6}+43 \mathbf{e}_{7}, 72 \mathbf{e}_{7}$ span the lattice of translations.

We need the following three theorems for proving our main result, which concerns crosses or semicrosses whose arm length may be greater than one.

THEOREM 3.3 [1, THEOREM 4, p. 324]. Assume that $j$ is relatively prime to $2 k n+1$ for $1 \leq j \leq k$. The finite abelian group $G$ is partitionable in the form

$$
G=\{0\} \cup\left(\bigcup_{i=1}^{n}\left\{g_{i}, 2 g_{i}, \ldots, k g_{i},-g_{i},-2 g_{i}, \ldots,-k g_{i}\right\}\right)
$$

if and only if for each prime divisor $p$ of $|G|$ the cyclic group $C$ of order $p$ is partitionable in the form

$$
C=\{0\} \cup\left(\bigcup_{i=1}^{t}\left\{c_{i}, 2 c_{i}, \ldots, k c_{i},-c_{i},-2 c_{i}, \ldots,-k c_{i}\right\}\right) .
$$

THEOREM 3.4 [2, THEOREM 3.3 , p. 218]. Assume that $j$ is relatively prime to $2 k n+1$ for $1 \leq j \leq k$ and the finite abelian $G$ is partitionable in the form (3.2). Then $G$ is partitionable in this form such that a set of generators of $G$ is a subset of $\left\{g_{1}, \ldots, g_{n}\right\}$.

THEOREM 3.5 [1, ThEOREM 1, p. 322]. Let $H, A$ be subsets and let $B$ be $a$ subgroup of an abelian group. Assume that $H=A+B$ is a factorization and that $A$ and $B$ are partitionable in the forms

$$
\begin{aligned}
& A=\{0\} \cup\left(\bigcup_{i=1}^{u}\left\{a_{i}, 2 a_{i}, \ldots, k a_{i},-a_{i},-2 a_{i}, \ldots,-k a_{i}\right\}\right), \\
& B=\{0\} \cup\left(\bigcup_{i=1}^{v}\left\{b_{i}, 2 b_{i}, \ldots, k b_{i},-b_{i},-2 b_{i}, \ldots,-k b_{i}\right\}\right),
\end{aligned}
$$

and that $j$ is relatively prime to $|B|$ for $1 \leq j \leq k$. Then $H$ is partitionable in the form

$$
H=\{0\} \cup\left(\bigcup_{i=1}^{w}\left\{h_{i}, 2 h_{i}, \ldots, k h_{i},-h_{i},-2 h_{i}, \ldots,-k h_{i}\right\}\right),
$$

where $\left\{h_{1}, \ldots, h_{w}\right\}=\left\{b_{i}: 1 \leq i \leq v\right\} \cup\left\{a_{i}+b: 1 \leq i \leq u, b \in B\right\}$.

THEOREM 3.6. If there exists an integer lattice tiling by $(k, n)$-crosses and if $2 k n+1$ has $s$ distinct prime divisors $(2 k n+1$ is the product of $s$ relatively prime 
powers) and if $j$ is relatively prime to $2 k n+1$ for $1 \leq j \leq k$, then there exists a rational lattice tiling by $(k, n)$-crosses in which one of the lattice vectors has $s-1$ noninteger coordinates.

Proof. According to Theorem 2.3, it is sufficient to show that there exists an abelian group $G$ which is factorizable in the form $(2.1)$ such that $q_{1}>1, \ldots, q_{s-1}>$ 1 , and $g_{n}$ generates $G$. Let $q_{1}, \ldots, q_{n}, r_{1}, \ldots, r_{s}$ be pairwise relatively prime positive integers, such that $q_{s}=\cdots=g_{n}=1$, and the others are greater than one, and $r_{1} \cdots r_{s}=2 k n+1$. Let $G$ be the cyclic group of order $(2 k n+1) q_{1} \cdots q_{s-1}$ and let $g$ be one of its generators. We shall use this group $G$ and choice of $q_{i}$ 's to prove the theorem.

When the set $\tau_{O}$ is a $(k, n)$-cross, then

$T\left(q_{1} g_{1}, \ldots, q_{n} g_{n}\right)^{*}=\{0\} \cup\left(\bigcup_{i=1}^{n}\left\{q_{i} g_{i}, 2 g_{i} g_{i}, \ldots, k q_{i} g_{i},-q_{i} g_{i},-2 q_{i} g_{i}, \ldots,-k q_{i} g_{i}\right\}\right)$.

Since there exists an integer lattice tiling by $(k, n)$-crosses, we conclude from Theorem 2.1 that there is an abelian group $G^{\prime}$ of order $2 n k+1$ such that $G^{\prime}=$ $T\left(g_{1}^{\prime}, \ldots, g_{n}^{\prime}\right)^{*}$; in other words $G^{\prime}$ is partitionable in the form

$$
G^{\prime}=\{0\} \cup\left(\bigcup_{i=1}^{n}\left\{g_{i}^{\prime}, 2 g_{i}^{\prime}, \ldots, k g_{i}^{\prime},-g_{i}^{\prime}, \ldots,-k g_{i}^{\prime}\right\}\right) .
$$

Consider the factor group $G /\left(r_{1} G\right)$, which is a cyclic group of order $r_{1}$. Since $2 k n+1$ is divisible by $r_{1}$, according to Theorem 3.3 the factor group is partitionable in the form

$$
r_{1} G \cup\left(\bigcup_{i=1}^{t}\left\{d_{i} h, 2 d_{i} h, \ldots, k d_{i} h,-d_{i} h,-2 d_{i} h, \ldots,-k d_{i} h\right\}\right),
$$

where $h$ is a generator of $G / r_{1} G$ and $d_{i} \in Z$. According to Theorem 3.4 we may assume that $d_{1}=1$. Let

$$
A=\{0\} \cup\left(\bigcup_{i=1}^{t}\left\{d_{i} g, 2 d_{i} g, \ldots, k d_{i} g,-d_{i} g,-2 d_{i} g, \ldots,-k d_{i} g\right\}\right) .
$$

By (3.3) the set $\{0\} \cup\left\{j d_{i}: 1 \leq|j| \leq k, 1 \leq i \leq t\right\}$ is a complete set of residues modulo $r_{1}$ and so $A$ is a complete set of coset representatives for $G$ modulo $r_{1} G$. Thus $G$ has a factorization $G=A+r_{1} G$. Since the $r_{i}$ 's and $q_{i}$ 's are pairwise relatively prime, the group $r_{1} G$, whose order is $r_{2} \cdots r_{s} q_{1} \cdots q_{s-1}$, is the direct sum of the cyclic groups $G_{1}, \ldots, G_{s-1}$ of orders $q_{1} r_{2}, q_{2} r_{3}, \ldots, q_{s-1} r_{s}$ respectively. If $\gamma_{i}$ is a generator of $G_{i}$, then $G_{i}$ is factorizable in the form $G_{i}=\left[\gamma_{i}\right]_{q_{i}}+q_{i} G_{i}$. Obviously, $q_{i} G_{i}$ is a cyclic group of order $r_{i+1}$ and $q_{i} \gamma_{i}$ is its generator. Since $2 k n+1$ is divisible by $r_{i+1}$, according to Theorem 3.3 the group $q_{i} G_{i}$ is partitionable in the form

$$
q_{i} G_{i}=\{0\} \cup\left(\bigcup_{j=1}^{t_{i}}\left\{\delta_{i j}, 2 \delta_{i j}, \ldots, k \delta_{i j},-\delta_{i j},-2 \delta_{i j}, \ldots,-k \delta_{i j}\right\}\right),
$$

where, according to Theorem 3.4 , we may assume that $\delta_{i 1}=q_{i} \gamma_{i}$. Finally, $G$ has the factorization

$$
G=A+q_{1} G_{1}+\cdots+q_{s-1} G_{s-1}+\left[\gamma_{1}\right]_{q_{1}}+\cdots+\left[\gamma_{s-1}\right]_{q_{s-1}} .
$$


According to Theorem 3.5, the set $A+q_{1} G_{1}+\cdots+q_{s-1} G_{s-1}$ is partitionable in the form

$$
\{0\} \cup\left(\bigcup_{i=1}^{n}\left\{\alpha_{i}, 2 \alpha_{i}, \ldots, k \alpha_{i},-\alpha_{i},-2 \alpha_{i}, \ldots,-k \alpha_{i}\right\}\right)
$$

and we may assume that $\alpha_{1}=q_{1} \gamma_{1}, \ldots, \alpha_{s-1}=q_{s-1} \gamma_{s-1}, \alpha_{n}=g$. Thus by choosing $g_{i}=\gamma_{i}, 1 \leq i \leq s-1$, and $g_{j}=\alpha_{j}, s \leq j \leq n, G$ has a factorization in the form (2.1) and $g_{n}=g$ generates $G$.

A similar method can be used to prove the following analogue of Theorem 3.6.

THEOREM 3.7. If there exists an integer lattice tiling by $(k, n)$-semicrosses, if $k n+1$ has $s$ distinct prime divisors and if $j$ is relatively prime to $k n+1$ for $1 \leq j \leq k$, then there exists a rational lattice tiling by $(k, n)$-semicrosses in which one of the lattice vectors has $s-1$ noninteger coordinates.

REMARK 3.1. In the proof of Theorem 3.6 we have used only the fact that the numbers $q_{1} r_{2}, \ldots, q_{s-1} r_{s}$ are pairwise relatively prime. So when $2 k n+1$ has $s$ distinct prime divisors and at least one of them occurs at least twice in the prime factorization of $2 k n+1$, the method of the proof of Theorem 3.6 gives $s$ noninteger coordinates.

We shall treat the example of $(2,37)$-semicross to illustrate the method of the proof of Theorem 3.7 and Remark 3.1. Now $k n+1=2 \cdot 37+1=3 \cdot 5^{2}$, so $k n+1$ has two distinct prime divisors 3 and 5 . Let $r_{1}=5, r_{2}=3, r_{3}=5, q_{1}=3, q_{2}=2$ and let $G$ be the cyclic group of order $r_{1} r_{2} r_{3} q_{1} q_{2}=450$. The numbers $q_{1} r_{2}$ and $q_{2} r_{3}$ are relatively prime. Let $g$ be a generator of $G$. The factor group $G / 5 G$ is factorizable in the form $5 G \cup\{g+5 G, 2 g+5 G\} \cup\{4 g+5 G, 8 g+5 G\}$ so $G$ is expressible in the form $(\{0\} \cup\{g, 2 g\} \cup\{4 g, 8 g\})+5 G$. The group $5 G$ is the direct sum of the groups $50 G$ and $45 G$. Obviusly, $50 G=[50 g]_{3}+150 G$ and $45 G=[45 g]_{2}+90 G$ are factorizations. The groups $150 G$ and $90 G$ are partitionable in the forms $\{0\} \cup\{150 g, 300 g\}$ and $\{0\} \cup\{90 g, 180 g\} \cup\{360 g, 720 g\}$. Finally, $G$ is expressible in the form

$$
G=\left[\{0\} \cup\left(\bigcup_{i=1}^{37}\left\{q_{i} g_{i}, 2 q_{i} g_{i}\right\}\right)\right]+\left[g_{1}\right]_{q_{1}}+\cdots+\left[g_{37}\right]_{q_{37}},
$$

where $\left\{q_{1} g_{1}, q_{2} g_{2}, \ldots, q_{37} g_{37}\right\}=\{150 g, 90 g, \ldots, g\}$ and $q_{1}=3, q_{2}=2, q_{3}=\cdots=$ $q_{37}=1$ so $g_{1}=50 g, g_{2}=45 g, \ldots, g_{37}=g$. According to Theorem 2.1 there exists a corresponding lattice tiling by $(2,37)$-semicrosses. Clearly $(-1 / 3) \mathbf{e}_{1}+50 \mathbf{e}_{37}$ and $(-1 / 2) \mathbf{e}_{2}+45 \mathbf{e}_{37}$ are vectors in this lattice tiling.

If in the prime factorization of $2 k n+1$ the exponents of some primes are greater than one, then the following theorem may provide more noninteger coordinates than Theorem 3.6 does.

THEOREM 3.8. If there exists an integer lattice tiling by $(k, n)$-crosses and $2 k n+1$ is the product of $s$ primes and if $j$ is relatively prime to $2 k n+1$ for $1 \leq$ $i \leq k$, then there exists a rational lattice tiling by $(k, n)$-crosses having noninteger coordinates on $m$ axes, where $m$ is the integer part of $s / 2$.

ProOF. Denote by $m$ the integer part of the half of the number of prime factors $2 k n+1$. Divide the $s$ prime factors of $2 k n+1$ into $m$ couples in case $s$ is even and divide the primes into $m-1$ couples and one triplet in case $s$ is odd. Denote by 
$r_{1}, \ldots, r_{m}$ the products of the elements of the couples and the triplet respectively. Let $r_{i}=u_{i} v_{i}$, where $u_{i}$ and $v_{i}$ are integers greater than one, for $1 \leq i \leq m$. Choose the integers $q_{1}, \ldots, q_{n}$ in such a way that $q_{1}>1, \ldots, q_{m}>1, q_{m+1}=\cdots=q_{n}=$ 1. Let $G$ be the internal direct sum of the cyclic groups $G_{1}, \ldots, G_{m}$ of orders $r_{1} q_{1}, \ldots, r_{m} q_{m}$ and generators $\gamma_{1}, \ldots, \gamma_{m}$, respectively. Consider the factor group $H=G /\left(\sum_{i=1}^{m} u_{i} G_{i}\right)$. In the well-known way we conclude that $H$ is partitionable in the form

$$
H=\{0\} \cup\left(\bigcup_{i=1}^{t}\left\{\chi_{i}, 2 \chi_{i}, \ldots, k \chi_{i},-\chi_{i},-2 \chi_{i}, \ldots,-k \chi_{i}\right\}\right),
$$

and we may assume that $\chi_{1}, \ldots, \chi_{m}$ generate $H$. Let $\varphi: G \rightarrow H$ be a homomorphism given by

$$
\left(\sum_{i=1}^{n} z_{i} \gamma_{i}\right) \varphi=\sum_{i=1}^{n} z_{i} v_{i} q_{i} \gamma_{i}, \quad z_{i} \in Z .
$$

Select $a_{i} \in G$ such that $a_{i} \varphi=\chi_{i}$ for $1 \leq i \leq m$ and let

$$
A=\{0\} \cup\left(\bigcup_{i=1}^{t}\left\{a_{i}, 2 a_{i}, \ldots, k a_{i},-a_{i},-2 a_{i}, \ldots,-k a_{i}\right\}\right) .
$$

Note that $G=A+\sum_{i=1}^{m} u_{i} G_{i}$ is a factorization. Using the factorizations $u_{i} G_{i}=$ $\left[u_{i} \gamma_{i}\right]_{q_{i}}+u_{i} q_{i} G_{i}$ we have the factorization

$$
G=A+\sum_{i=1}^{m} u_{i} q_{i} G_{i}+\sum_{i=1}^{m}\left[u_{i} \gamma_{i}\right]_{q_{i}}
$$

According to Theorem 3.5 the set $A+\sum_{i=1}^{m} u_{i} q_{i} G_{i}$ is partitionable in the form

$$
\{0\} \cup\left(\bigcup_{j=1}^{n}\left\{\alpha_{j}, 2 \alpha_{j}, \ldots, k \alpha_{j},-\alpha_{j},-2 \alpha_{j}, \ldots,-k \alpha_{j}\right\}\right)
$$

and we may assume that $\alpha_{j}=u_{j} q_{j} \gamma_{j}$ and $\alpha_{n-j+1}=\gamma_{j}, 1 \leq j \leq m$. If we select $g_{i}=u_{i} \gamma_{i}, 1 \leq i \leq m$, and $g_{j}=\alpha_{j}, m+1 \leq j \leq n$, then $G$ is expressible in the form (2.1). So according to Theorem 2.1 there exists a rational lattice tiling by $(k, n)$-crosses. Now we shall prove that the set

$$
S_{i}=\left(A+\sum_{j=1}^{m} u_{j} q_{j} G_{j}\right)+\sum_{\substack{j=1 \\ j \neq i}}^{m}\left[g_{j}\right]_{q_{j}}=T\left(q_{1} g_{1}, \ldots, q_{n} g_{n}\right)^{*}+\sum_{\substack{j=1 \\ j \neq i}}^{m}\left[g_{j}\right]_{q_{j}}
$$

is not a subgroup of $G$ for $1 \leq i \leq m$. Indeed, the set $\left\{\gamma_{1}, \ldots, \gamma_{m}\right\} \subseteq S_{i}$ generates a group of order

$$
(2 k n+1) q_{1} \cdots q_{m}>\left(1 / q_{i}\right)(2 k n+1) q_{1} \cdots q_{m}=\left|S_{i}\right| .
$$

The proof of the following theorem is carried out analogously to the proof of the preceding theorem. 
THEOREM 3.9. If there exists an integer lattice tiling by $(k, n)$-semicrosses, if $k n+1$ is a product of $s$ primes and if $i$ is relatively prime to $k n+1$ for $1 \leq i \leq k$, then there exists a rational lattice tiling by $(k, n)$-semicrosses having noninteger coordinates on $m$ axes, where $m$ is the integer part of $s / 2$.

Consider the example of the $(2,40)$-semicross to illustrate the method of the proof of Theorem 3.9. Now $k n+1=2 \cdot 40+1=3^{4}$. Let $G$ be the direct sum of the cyclic groups $G_{1}$ and $G_{2}$ of order 18 and generators $\gamma_{1}$ and $\gamma_{2}$, respectively. The factor group $G /\left(3 G_{1}+3 G_{2}\right)$ is partitionable in the form

$3 G_{1}+3 G_{2} \cup\left\{\chi_{1}, 2 \chi_{1}\right\} \cup\left\{\chi_{2}, 2 \chi_{2}\right\} \cup\left\{\chi_{1}+\chi_{2}, 2\left(\chi_{1}+\chi_{2}\right)\right\} \cup\left\{\chi_{1}+2 \chi_{2}, 2\left(\chi_{1}+2 \chi_{2}\right)\right\}$, where $j \chi_{i}=j \gamma_{i}+3 G_{1}+3 G_{2}, 1 \leq i, j \leq 2$. Let

$$
A=\{0\} \cup\left\{\gamma_{1}, 2 \gamma_{1}\right\} \cup\left\{\gamma_{2}, 2 \gamma_{2}\right\} \cup\left\{\gamma_{1}+\gamma_{2}, 2\left(\gamma_{1}+\gamma_{2}\right)\right\} \cup\left\{\gamma_{1}+2 \gamma_{2}, 2\left(\gamma_{1}+2 \gamma_{2}\right)\right\} \text {. }
$$

Since $3 G_{1}=\left\{0,3 \gamma_{1}\right\}+\left\{0,6 \gamma_{1}, 12 \gamma_{1}\right\}$ and $3 G_{2}=\left\{0,3 \gamma_{2}\right\}+\left\{0,6 \gamma_{2}, 12 \gamma_{2}\right\}$, we have $G=A+3 G_{1}+3 G_{2}+\left[3 \gamma_{1}\right]_{2}+\left[3 \gamma_{2}\right]_{2}$. Thus $G$ is expressible in the form

$$
G=\left[\{0\} \cup\left(\bigcup_{i=1}^{40}\left\{q_{i} g_{i}, 2 q_{i} g_{i}\right\}\right)\right]+\left[g_{1}\right]_{q_{1}}+\cdots+\left[g_{40}\right]_{q_{40}}
$$

where $q_{1}=q_{2}=2, q_{3}=\cdots=q_{40}=1$ and $g_{1}=3 \gamma_{1}, g_{2}=3 \gamma_{2}, \ldots, g_{39}=\gamma_{1}$, $g_{40}=\gamma_{2}$.

ACKNOWLEDGEMEnTs. The author is indebted to the referee for pointing out the phenomenon given by Remark 2.4 and for other valuable suggestions.

\section{REFERENCES}

1. W. Hamaker and S. K. Stein, Splitting groups by integers, Proc. Amer. Math. Soc. 46 (1974), $322-324$.

2. S. Szabó, Rational tilings by n-dimensional crosses, Proc. Amer. Math. Soc. 87 (1983), 213-222.

Department of Civil Engineering Mathematics, Technical University BUdAPEST, 1111 BUDAPEST, STOCZEK U. 2, HUNGARY 